\title{
Transverse limb defect: a case report
}

\section{Vidyashree Ganesh Poojari*, Muralidhar V. Pai, Akhila Vasudeva, Jayaraman Nambiar}

Department of Obstetrics \& Gynecology, KMC Manipal, Manipal University, Manipal, Karnataka, India

\author{
Received: 29 March 2015 \\ Revised: 06 April 2015 \\ Accepted: 09 May 2015
}

\author{
*Correspondence: \\ Dr. Vidyashree Ganesh Poojari, \\ E-mail: drvidyaganesh@gmail.com
}

Copyright: (c) the author(s), publisher and licensee Medip Academy. This is an open-access article distributed under the terms of the Creative Commons Attribution Non-Commercial License, which permits unrestricted non-commercial use, distribution, and reproduction in any medium, provided the original work is properly cited.

\begin{abstract}
Transverse limb defects are a relatively rare problem (3.5-6.9/10000 births) with a fairly low prenatal detection rate (55\%). The possible etiology for this condition could be as part of a genetic condition or amniotic band syndrome. The detection rate can be improved with the use of careful anatomical survey during anomaly scan. In this case report, transverse limb defect was detected in the second trimester.
\end{abstract}

Keywords: Transverse limb defect, Prenatal diagnosis, Termination of pregnancy

\section{INTRODUCTION}

The incidence of limb reduction defects varies from 3.56.9 per 10000 births. The prenatal detection rate is approximately $55 \%$. However, this varies considerably depending on the application of imaging guidelines, and on the population studied. The defects range from complete absence of limbs (Amelia) to partial absence (meromelia). The commonest limb reduction defect is a terminal transverse defect, which is usually unilateral, isolated, and sporadic in occurrence. This is defined as the partial or complete absence of one or more fetal limbs beyond a certain point, leaving a stump, and is usually categorized according to the last remaining bone segment. This case report describes an isolated transverse limb defect which was detected in the second trimester targeted scan.

\section{CASE REPORT}

A 28 years, primigravida at 18 weeks, was referred to the fetal medicine unit for a target scan. The first trimester scan done at 11 week showed a single live intrauterine gestation with a CRL of $48 \mathrm{~mm}$ corresponding to the period of amenorrhea. The NT was $1.8 \mathrm{~mm}$.
A targeted ultrasound was performed and was consistent with biometry of 18 weeks. The placenta, liquor and fetal activity were normal. On detailed targeted imaging, the left humerus was normal in length and the elbow was imaged. Distal to the elbow, the radius and ulna were seen for a length of 1 to $1.5 \mathrm{~mm}$ and were absent beyond that length (Figure 1). The hand was not visualised. No attachment of amniotic membrane to the distal segment of the upper limb was noted on careful observation. Movements and muscle mass was normal in the proximal segment. The right upper limb was imaged and confirmed to be normal in the proximal, mid and distal segments. Movements of the right upper limb were also normal in shoulder and elbow joints. Hand opening and closure was also seen. The lower limbs were structurally normal in the proximal, mid and distal segments and movements were seen. The other fetal structures and Fetal ECHO was normal.

The patient and her family were counseled in detail. The possibilities of prosthesis for the left limb and that the other limb was normal in function thus could be used for writing etc. were emphasized and discussion with plastic surgeons was offered. However the couple decided that they could not raise a child with physical disability. After 
the second counseling, termination of pregnancy was opted for.

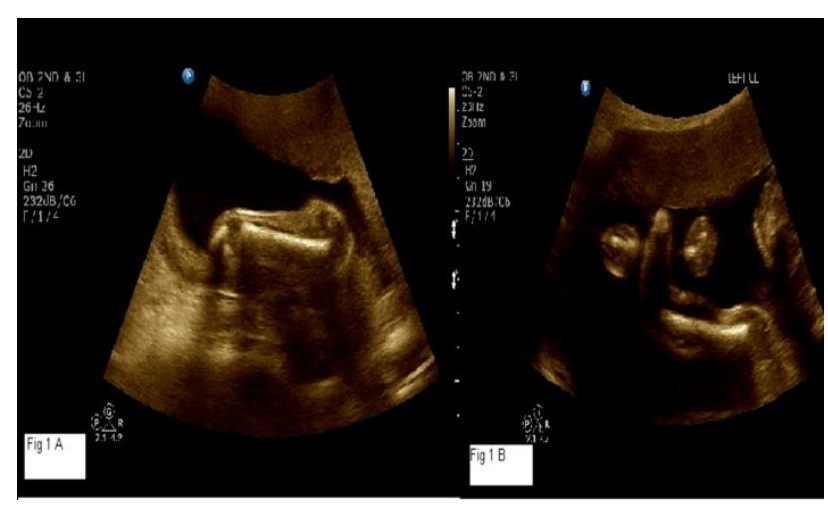

Figure 1: Ultrasound pictures - A) Shows the rudimentary forearm bones, radius and ulna. B)

Ultrasound of the right upper limb showing normally developed proximal segment with humerus and a blunt ending distal segment. Forearm has not developed beyond $2 \mathrm{~cm}$.

The patient was induced with 4 doses of $100 \mathrm{mcg}$ PG E1 tablets $4^{\text {th }}$ hourly per vaginum and expelled the fetus. The fetus weighed 357 grams, and showed transverse defect of the left forearm. The right upper limb was normal (Figure 2) and there were no other external anomalies. The patient was discharged from hospital after two days. The couple accepted for autopsy of the fetus. The couple was counseled that the recurrence rates of transverse limb defects is very low due to its sporadic occurrence. Prepregnancy folic acid and first trimester scan in a referral center was advised.

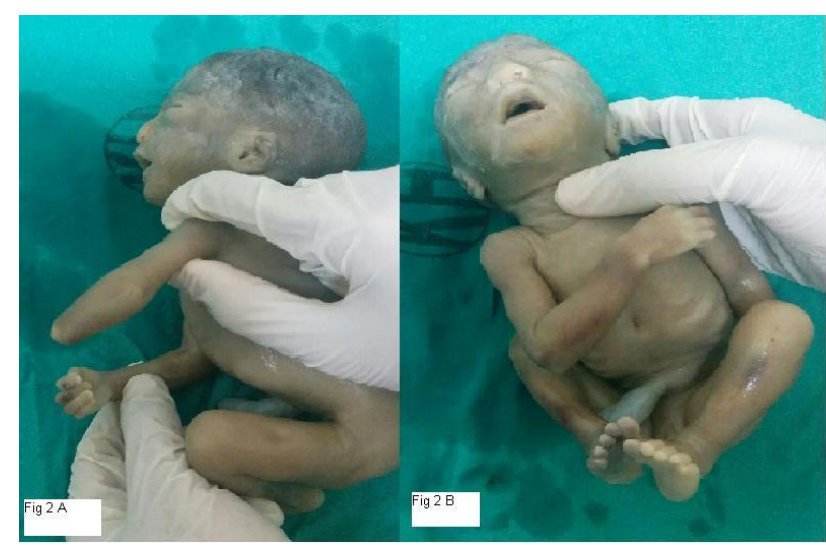

Figure 2: Autopsy pictures - A) Showing a transverse limb defect affecting one of the upper limbs. B) Showing normally developed other limbs, with no obvious features suggestive of amniotic bands/early amniotic rupture.

\section{DISCUSSION}

Most cases of limb defects are believed to be secondary to vascular insult occurring very early in embryonic life or amnion rupture sequence. Amnion rupture sequence causes formation of amniotic bands which can cause many different scenarios. A study of 1010 pre-viable fetuses (9-20 weeks developmental age) was performed to determine the result of amnion rupture sequence. 18 fetuses were affected with the incidence of 1:56. Eleven fetuses had limb constrictions and amputations only; 7 fetuses also had non-limb involvement, such as encephalocele, unusual facial clefts, and abdominal defects. In 6 pregnancies, constrictions of the umbilical cord by amniotic bands were the cause of fetal intrauterine death.

Transverse limb defects can also be caused by ingestion of teratogens such as misoprostol which may be used for first trimester termination of pregnancy. ${ }^{1}$ They can also be caused by maternal ingestion of vasospastic drugs and maternal diabetes, smoking and ingestion of alcohol. ${ }^{2}$ Amniotic band syndrome in the second trimester has been reported following diagnostic amniocentesis but its spontaneous occurrence is rare.

This case is reported to emphasize the importance of first trimester scan which should be carefully performed according to standard guidelines. ${ }^{3}$ The guidelines suggest demonstrating four limbs with three segments and as an option recommend demonstration of normal orientation of hands and feet.

The practice of demonstrating both the humerii and then both bones of the forearms, followed by the coronal section of the fetal hand demonstrating the open fingers, helps greatly in improving detection rates not only of larger transverse limb reduction defects but also of reduction of fingers, ectrodactyly and polydactyly. First trimester screening for anomalies is now standard of care and performed with trans-abdominal or trans-vaginal route to obtain the necessary images. ${ }^{4,5}$ Early detection provides the patient with a choice of termination of pregnancy at an earlier gestational age, reducing the physical morbidity and to some degree the emotional morbidity associated with the procedure. Although first trimester scanning cannot be relied on exclusively as a targeted scan, it does have the potential to detect many anomalies early thus benefiting the patient.

Funding: No funding sources

Conflict of interest: None declared

Ethical approval: Not required

\section{REFERENCES}

1. Genest DR, Di Salvo D, Rosenblatt MJ, Holmes LB. Terminal transverse limb defects with tethering and omphalocele in a 17 week fetus following first trimester misoprostol exposure. Clin Dysmorphol. 1999;8(1):53-8.

2. Saeed F, Paramasivam G, Wiechec M, Kumar S. Incidence and outcome of antenatally diagnosed isolated transverse limb defects. Ultrasound Obstet Gynecol. 2009;34(Suppl 1):177-284. 
3. Salomon LJ, Alfirevic Z, Bilardo CM, Chalouhi GE, Ghi T, Kagan KO, et al. ISUOG Practice Guidelines: performance of first-trimester fetal ultrasound scan. Ultrasound Obstet Gynecol. 2013;41:102-13.

4. Cullen MT, Green J, Whetham J, Salfia C, Gabrielli S, Hobbins JC. Transvaginal ultrasonographic detection of congenital anomalies in the first trimester. Am J Obstet Gynecol. 1990;163:466-76.
5. Achiron R, Tadmor O. Screening for fetal anomalies during the first trimester of pregnancy: transvaginal versus transabdominal sonography. Ultrasound Obstet Gynecol. 1991;1:186-91.

DOI: $10.18203 / 2320-1770 . i j r \operatorname{cog} 20150124$

Cite this article as: Poojari VG, Pai MV, Vasudeva A, Nambiar J. Transverse limb defect: a case report. Int J Reprod Contracept Obstet Gynecol 2015;4:902-4. 